\title{
Statistical Identification and Analysis of Defect Development in Digital Imagers
}

\author{
Jenny Leung ${ }^{\mathrm{a}}$, Glenn H. Chapman ${ }^{* a}$, Zahava Koren ${ }^{\mathrm{b}}$, Israel Koren ${ }^{\mathrm{b}}$ \\ ${ }^{a}$ School of Engineering Science, Simon Fraser University, \\ Burnaby, BC, Canada, V5A 1S6 \\ ${ }^{\mathrm{b}}$ Dept. of Electrical \& Computer Engineering, University of Massachusetts, \\ Amherst, MA, USA 01003
}

\begin{abstract}
The lifetime of solid-state image sensors is limited by the appearance of defects, particularly hot-pixels, which we have previously shown to develop continuously over the sensor lifetime. Analysis based on spatial distribution and temporal growth of defects displayed no evidence of the defects being caused by material degradation. Instead, high radiation appears to accelerate defect development in image sensors. It is important to detect these faulty pixels prior to the use of image enhancement algorithms to avoid spreading the error to neighboring pixels. The date on which a defect has first developed can be extracted from past images. Previously, an automatic defect detection algorithm using Bayesian probability accumulation was introduced and tested. We performed extensive testing of this Bayes-based algorithm by detecting defects in image datasets obtained from four cameras. Our results have indicated that the Bayes detection scheme was able to identify all defects in these cameras with less than $3 \%$ difference from visual inspected result. In this paper, we introduce an alternative technique, the Maximum Likelihood detection algorithm, and evaluate its performance using Monte Carlo simulations based on three criterias: image exposure, defect parameters and pixel estimation. Preliminary results show that the Maximum likelihood detection algorithm is able to achieve higher accuracy than the Bayes detection algorithm, with $90 \%$ perfect detection in images captured at long exposures $(>0.125 \mathrm{~s})$.
\end{abstract}

Keywords: defect detection, hot pixel, imager defects, active pixel sensor, APS, CMOS image sensor, demosaicing

\section{INTRODUCTION}

Like all microelectronic devices, digital imagers develop defects while operating in the field. Advanced digital cameras with high pixel density and enhanced image processing functions improve image quality; however, little work has been done to address the problem of accumulation of defective pixels in sensors. The effect of these faulty pixels, if not corrected, can spread to the neighboring pixels through the use of image enhancing algorithms (such as demosaicing) and thus shorten the useful lifetime of the sensor.

In-field defects are permanent and their number increases continuously over the sensor lifetime. Although camera defects can be handled by factory recalibration, this is a time consuming and expensive process, and not feasible for sensors in most embedded applications. By acquiring more knowledge about the source and characteristics of faulty pixels, an in-field defect correction scheme can be developed which will prolong the sensor lifetime.

In our recent study ${ }^{1,2,3}$ we applied manual calibration techniques to identify types of in-field defects in a large number of semi-professional cameras. Despite the difference in sensor technology between Charge Couple Device (CCD) and Active Pixel Sensors (APS), our study has shown that both types of image sensors will develop defects over their lifetime. In fact, hot pixels are the dominant defect type observed in both types of sensors, although as we have shown ${ }^{1}$, hot pixels with significant offsets often appear as partially stuck or stuck high defects. In our analytical study ${ }^{3}$, we

* glennc@ensc.sfu.ca; phone +1-778-782-3814; fax +1-778-782-4951

Digital Photography V, edited by Brian G. Rodricks, Sabine E. Süsstrunk,

Proc. of SPIE-IS\&T Electronic Imaging, SPIE Vol. 7250, 72500W · C 2009 SPIE-IS\&T

CCC code: 0277-786X/09/\$18 · doi: 10.1117/12.806109 
investigated both the spatial distribution and the temporal growth of in-field defects. We have shown that defects are randomly distributed over the sensor area, suggesting that the defect source is not due to materials related failures. In fact, similar to the results presented in another study ${ }^{4}$, we observed that cameras that have been on several transatlantics/pacific flights experienced a higher defect rate, indicating that high radiation might be the main defect source. By tracing the defect first development date for every identified defective pixel, we can gain better insight into how defects develop and compare defect growth rates among different sensors.

In this paper we expand our previous analytical study using additional defect datasets and the results are summarized in Section 2. Previously ${ }^{1}$, we have introduced a defect detection algorithm utilizing Bayesian statistics to trace the first appearance of a defect based on an image set. To further enhance the accuracy of the detection algorithm, in this paper we introduce a second methodology for identifying defect creation dates; a Maximum Likelihood detection algorithm. The development of the algorithm is discussed in Section 3, while the simulation and comparison of the two approaches are described in Section 4. Based on our recent work $^{2}$, we have applied the detection algorithm to the available image sets from several cameras. Extended results on defect growth from the new cameras are discussed in Section 5.

\section{DEFECT CHARACTERIZATION}

Different defect sources will cause different behavioral characteristics of defects. By identifying and analyzing spatial and temporal growth of in-field defects, we can gain a better insight into the defect source mechanisms in image sensors. From our on-going analysis of defects from a set of cameras, hot pixels were found to be the dominant in-field defects. Hot pixels can be identified using dark frame calibration where a set of images is captured under the absence of light at long exposure duration. The dark response of hot pixels is shown in Figure 1. As shown in plot (a) the dark response of traditional hot pixels has an illumination independent component that increases linearly with exposure setting. In our analysis we have also observed partially-stuck hot pixels as shown in plot (b) which have an additional offset that is independent of the capturing mode. The offset will cause the pixel to saturate at short exposures thus further reducing the dynamic range.

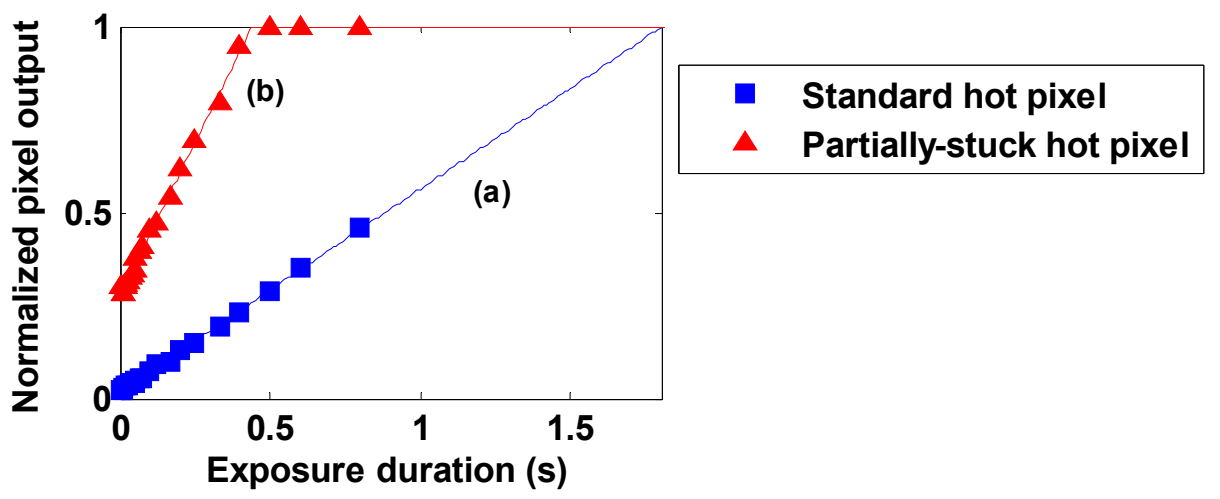

Figure 1: Comparing the dark response of a good pixel and a hot pixel.

The normalized response $f$ of any pixel is a function of photocurrent $I_{\text {photo }}$, exposure duration $T_{\text {exp }}$, and dark current $I_{\text {Dark }}$ as characterized by Equation (1) where an output of 1 represents saturated response. The phenomenon of hot pixels is defined by two main defect parameters: the dark current, which is much higher than regular levels, and the pixel offset, denoted by $b$. The product $m \cdot I_{\text {Dark }} \cdot T_{\exp }$ is the effective dark current integrated at the pixel output.

$$
f_{\text {Hot-Pixel }}\left(I_{\text {photo }}, I_{\text {Dark }}, T_{\exp }\right)=m \cdot\left(I_{\text {photo }} T_{\exp }+I_{\text {Dark }} T_{\exp }\right)+b
$$

In our most recent experiments we have observed additional defects in both old and new cameras. The breakdown of the hot pixels observed in each tested camera is summarized Table 1. As opposed to 98 defects from 10 cameras ${ }^{1}$, we now have observed a total of 170 defects from 14 cameras, and the fraction of partially-stuck hot pixels increased to $50 \%$. 
Hot pixels are most observable in long exposure images due to the additional exposure dependent component; however, partially stuck hot pixels are visible even in short exposure images due to the additional pixel offset. Results from calibration indicate that the offset of these partially stuck hot pixels can saturate at as high as $40 \%$ of the pixel dynamic range; these defects will manifest themselves in all capturing conditions.

Table 1: Summary of in-field defects from tested cameras.

\begin{tabular}{|c|c|ccc|c|}
\hline \multirow{2}{*}{ Camera } & & \multicolumn{4}{|c|}{ Number of defects found } \\
\cline { 2 - 6 } & \multirow{3}{*}{ Hot pixels } & Total \\
\hline A & Nears) & No offset & W/ offset & Total & \\
B & 4 & 0 & 11 & 11 & 11 \\
C & 3 & 0 & 5 & 5 & 5 \\
D & 2.5 & 6 & 5 & 11 & 11 \\
F & 1 & 0 & 7 & 7 & 7 \\
G & 5 & 26 & 0 & 26 & 26 \\
H & 2.5 & 17 & 0 & 17 & 17 \\
I & 0.5 & 0 & 2 & 2 & 2 \\
J & 2 & 2 & 0 & 3 & 3 \\
K & 3 & 4 & 17 & 19 & 19 \\
L & 3 & 9 & 1 & 31 & 31 \\
M & 9 & 0 & 2 & 10 & 10 \\
N & 2 & 17 & 1 & 2 & 2 \\
O & 4.5 & 1 & 7 & 8 & 18 \\
\hline
\end{tabular}

\subsection{Spatial distribution and temporal distribution of defects}

Both spatial and temporal distributions of defects can help in identifying the defect source. From our continuing study ${ }^{3}$, we derived the spatial distribution of defects by collecting the distances between defects on individual sensors. The results with our 14 tested cameras are summarized in a histogram plot in Figure 2a. The single peak and broad distribution of defect distances with an average of $10 \mathrm{~mm}$ separation between defects on these large area sensors $(24 \mathrm{~mm}$ x $32 \mathrm{~mm}$ ) indicates that there are no local defect clusters in any sensor. The fact that similar characteristics hold true for both CCD and APS sensors suggests that the defect source is not material degradation.

The rate at which defects grow in time is also important in defect analysis. For defects related to material degradation, we would expect the time between consecutive defects to get shorter. Instead, we observed that the number of defects grew linearly with time, indicating a constant growth rate. We next compared the defect growth rates for the two types of sensors. In Figure 2b, we plot the number of accumulated defects versus the camera age for each sensor type. We can see that for both senor types, defect development is a continuous process with an almost constant growth rate, resulting in a linear in time number of defects. CCD sensors, with a growth rate of 5.2 defects/year, appear to be more sensitive to defects than APS which exhibited a growth rate of 2.2 defects/year. 


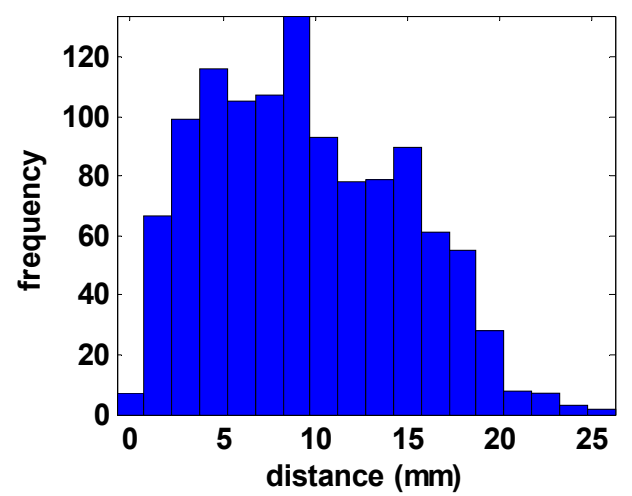

(a) Defect distance histogram for APS and CCD imagers

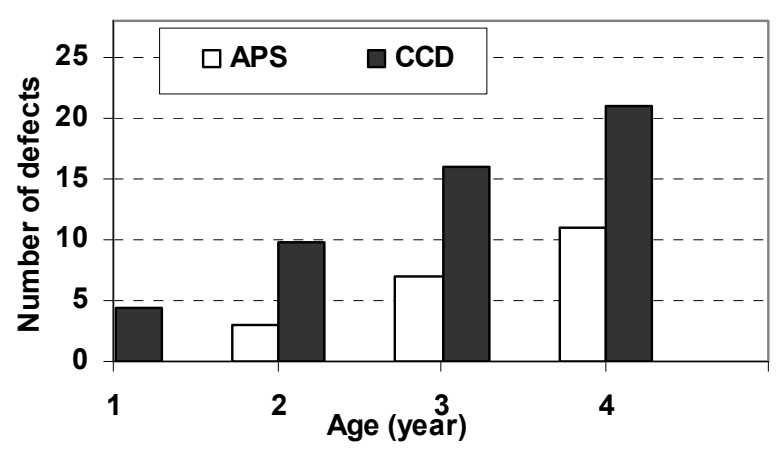

(b) Collective defect growth comparison

Figure 2: Defect distance and temporal growth analysis.

The observed in-field defects do not heal over time; once a pixel becomes defective it manifests itself as a faulty pixel in all subsequent images. Therefore, the individual defect growth rate for each camera can be traced by analyzing all of the historical images taken by this camera. The previously developed manual technique for extracting the defect occurrence time is slow and limited by the few available image sets (mainly due to privacy issues). To gain access to a larger data set, we have developed an automatic defect-tracing algorithm that detects the presence or absence of hot pixels in a collection of images taken by individual cameras. With the development of such an algorithm, we can gain access to a wider set of cameras by providing users with a simple tool that extracts data from their photos automatically.

\section{DEFECT TRACE ALGORITHM}

To analyze how defects develop in individual sensors, we need a technique to identify the date on which the defect first appeared. A useful characteristic of digital cameras is that every image captured by the camera is also a record of the current state of the sensor with image acquisition information (capture date, camera settings, etc). Therefore, the historical image collection from a camera provides information about the aging process of the image sensor. As shown in Figure 3, after identifying the spatial location of each defect we can search through the entire image dataset from this camera and pinpoint the image where the defect first appeared. The spatial location and hot pixel parameters can be easily extracted from the dark frame calibration. Defects can be identified by visual inspection; however, with a large image dataset (about $100 \mathrm{~Gb}$ ), this procedure is slow and, due to privacy issues, will limit our access to a wide range of datasets. In the following section we discuss two techniques to automate the defect detection procedure using statistical analysis.

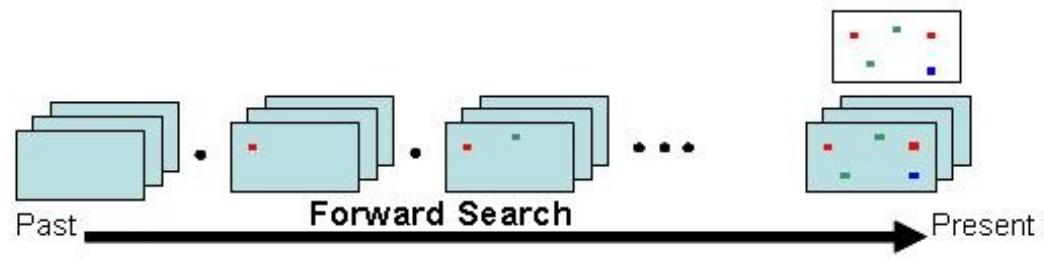

Figure 3: Defect detection procedure from a data set of images.

\subsection{Pixel Estimation}

In our algorithm, the imager is described by an array of $W \times H$ pixels and the output at pixel location $(i, j)$ is denoted by $y_{i, j}$. Most images are captured in color mode such that each color pixel is composed of three 8-bit values representing the three color channels (Red, Green, and Blue). The 8-bit value represents the intensity of each color channel, where 0 is dark and 255 is saturation. 
As shown in Section 2, hot pixels are the dominant in-field defects found in all tested cameras. Therefore, in our algorithm a pixel may be in one of the two states: good or hot. Assuming all pixels have a unity gain, the pixel characterization Equation (1) can be simplified to Equation (2) where the output of a good pixel measures the amount of incident light that strikes the pixel, denote by $x$. A hot pixel is characterized by the defect parameters $\left(T_{\text {exp }} \cdot I_{\text {Dark }}\right)+b$ and this can be treated as a dark offset denoted by $\Delta$.

$$
y=x+\left(T_{\exp } \cdot I_{\text {Dark }}\right)+b=x+\Delta
$$

We can detect the presence of hot pixels using adjacent pixel information. For any hot pixel, the output of the sensor will be higher than a non-defective pixel due to the additional signal of the dark current and offset. By interpolating the adjacent pixels we can calculate the expected value of the center pixel and estimate the probability that it is defective. Let $z_{i, j}$ denote the interpolated pixel value, and $y_{i, j}$ denote the actual pixel output value. Then, the error is defined as $e_{i, j}=y_{i, j}-z_{i, j}$. By interpolating throughout the image and collecting the image-wide interpolation errors, we can derive the Probability Distribution Function (PDF), $p_{E}(e)$, and the Cumulative Distribution Function (CDF), $P_{E}(e)$. The error $e_{i, j}$ measures the discrepancy between the expected and actual pixel value. Thus, for any good pixel, the error should be approximately zero; and will be close to $\Delta$ for a hot pixel. Given the expected value of the pixel, we compare this output to a good pixel model and a hot pixel model using the error value. To model a good pixel, we compare the actual pixel output with the expected value as shown in Equation (5), and the interpolation error PDF can provide a statistical measure on the likelihood of the pixel being good. Similarly we can model the pixel output as a hot pixel by subtracting the dark offset. Because the dark current fluctuates, we estimate this value with $P_{E}(e)$.

$$
\begin{gathered}
G\left(y_{k}\right)=\operatorname{Prob}\left(y_{k} \mid \operatorname{Good}\right)=p_{E}\left(y_{k}-z_{k}\right) \\
H\left(y_{k}\right)=\operatorname{Prob}\left(y_{k} \mid H o t\right)=\frac{1}{255-\Delta_{\min }+1} \cdot\left[P_{E}\left(y_{k}-z_{k}-\Delta_{\min }\right)-P_{E}\left(y_{k}-z_{k}-255\right)\right]
\end{gathered}
$$

The estimation on the presence or absence of defects depends on the interpolation error functions PDF $p_{E}(e)$ and CDF $P_{E}(e)$. Therefore, the choice of interpolation scheme will significantly affect the accuracy of the algorithm. The nearest neighboring pixels interpolation provides the most accurate estimation under the defect free condition. It is important to note that color images produced by cameras are processed by a set of internal algorithms that ignore the presence of defects. In particular, the demosaicing algorithm (color interpolation function) that is used to interpolate missing color channels in raw images, will spread a single defective pixel to the neighboring pixels as shown in Figure 5.

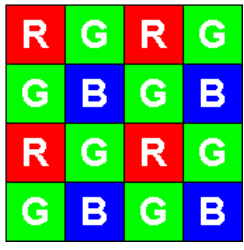

Figure 4. Raw CFA (RGGB) image pattern.

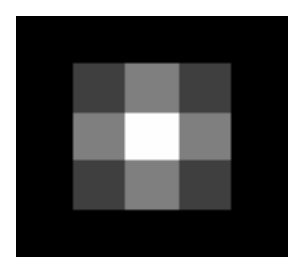

Figure 5. Demosaicing an image with a defect

Thus, in a colored image with a defective pixel, the nearest neighbors will not provide the best estimate of the expected pixel value. Being aware of the possible spreading of defects to neighboring pixels by demosaicing, we generate the pixel estimate using a ring averaging scheme shown in Figure 6, where we ignore all the pixels in the center and compute the pixel estimate using only the pixels located on the perimeter of the interpolation region. In our simulation, three interpolation schemes have been considered: $3 \times 3$ averaging, $5 \times 5$ and $7 \times 7$ ring averaging. 


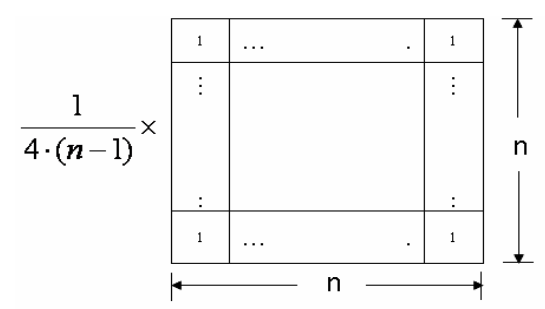

Figure 6. Ring averaging coefficient mask

\subsection{Bayesian Accumulation}

Pixel estimation provides a statistical measure for the status of the pixel based on a comparison between good and hot pixel models. However, the status of the pixel cannot be determined based solely on a single image analysis; thus, to accumulate the state of the pixel at the $k^{\text {th }}$ image, we evaluate the status of the pixel using Equations (5) and (6).

$$
\begin{gathered}
\operatorname{Prob}\left(\operatorname{Good} \mid y_{k}\right)=\frac{\operatorname{Prob}\left(y_{k} \mid \operatorname{Good}\right) \cdot \operatorname{Prob}\left(\operatorname{Good} \mid y_{k-1}\right)}{\operatorname{Prob}\left(y_{k} \mid \operatorname{Good}\right) \cdot \operatorname{Prob}\left(\operatorname{Good} \mid y_{k-1}\right)+\operatorname{Prob}\left(y_{k} \mid \operatorname{Hot}\right) \cdot \operatorname{Prob}\left(\operatorname{Hot} \mid y_{k-1}\right)} \\
\operatorname{Prob}\left(\operatorname{Hot} \mid y_{k}\right)=1-\operatorname{Prob}\left(\operatorname{Good} \mid y_{k}\right)
\end{gathered}
$$

For a good pixel we will obtain $\operatorname{Prob}\left(\operatorname{Good} \mid y_{k}\right) \approx 1$ while for a hot pixel $\operatorname{Prob}\left(\operatorname{Good} \mid y_{k}\right) \approx 0$; thus the change in the accumulated probability reflects the change in the state of the pixel. Figure 7 shows a typical plot of the probability $\operatorname{Prob}\left(\operatorname{Good} \mid y_{k}\right)$ evaluated after analyzing for a specific pixel location from a sequence of images for a camera. To estimate the first defect date from this result we apply a threshold test on the accumulated probability, for example, when $\operatorname{Prob}\left(\operatorname{Good} \mid y_{k}\right)$ drops below 0.5 this will identify the date of the first defect. However, as the plot in Figure 7 demonstrates, the accumulated probability has multiple transitions due to interpolation errors and the variations in the visibility of a hot pixel among images.

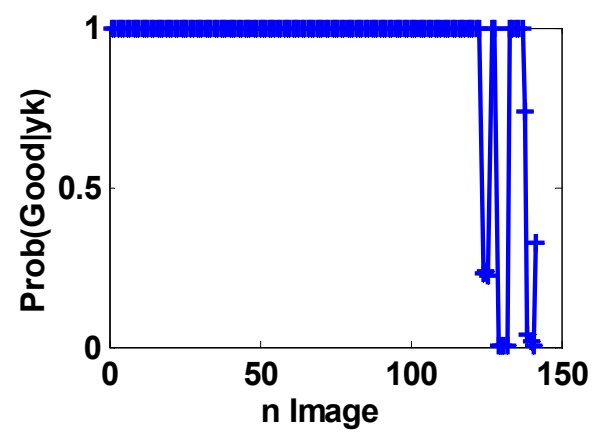

Figure 7. Plot of $\operatorname{Prob}\left(\operatorname{Good} \mid y_{k}\right)$ over a sequence of images.

In a previous study ${ }^{2}$, we have shown that the accuracy of pixel estimation is affected by the complexity of the particular image scene, ISO setting and shutter speed. Local regions in a fine detail image tend to have large color variation; thus pixel estimate in such local regions will have a large estimation error. Recall from Section 3.1 that pixel status evaluation is dependent on neighboring pixels; thus, large pixel estimation error will cause false detection. By evaluating the color mean and variance from the local region around the pixel of interest, we gain knowledge on the accuracy of pixel estimation within the local region. To reduce the detection error due to interpolation error, we can apply a post correction procedure. Given an accumulated probability result as shown in Figure 7, we apply the threshold test to identify the first potential defect date. For the image where the defect was first detected, we evaluate the color mean and variance within the confined region around the defective pixel. If the local color variance exceeds our preset limit, this indicates the detection is caused by an interpolation error, and thus, we discard the result and continue to the next potential defect date point. The algorithm flow is summarized in Figure 8. 


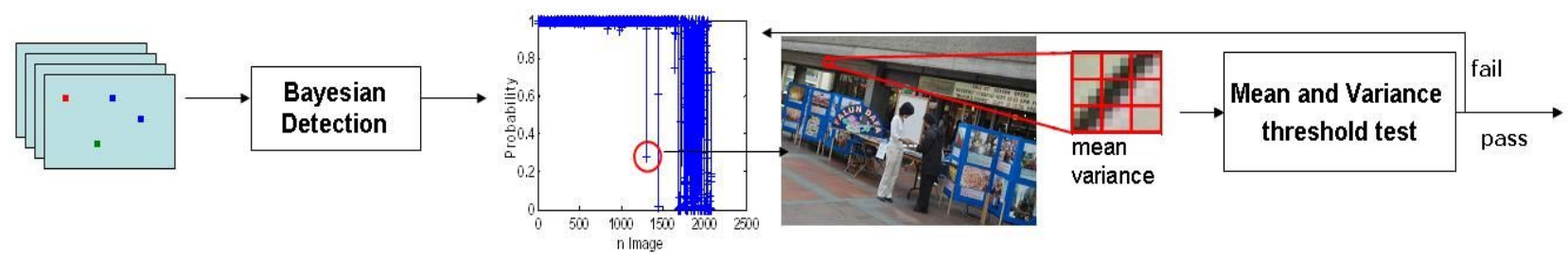

Figure 8. Local statistics correction procedure

As was shown in our previous $s_{\text {ady }}{ }^{2}$, false detection is reduced significantly with this additional local statistical correction, and the detection scheme achieves sufficient accuracy when applied to real image datasets. In Section 5 we examine the defect development dates for image collections from 4 tested cameras using both visual inspection and Bayes detection.

\subsection{Maximum Likelihood Detection}

Our previous statistical analysis ${ }^{3}$ of defect growth rate has suggested that defects develop at a constant rate. Let $n$ denote the number of images and let $y_{1}, y_{2}, \ldots, y_{m}, \ldots, y_{n}$ denote a certain pixel's outputs in these images with $k=1$ being the oldest image in the collection. If a defect appeared first in image $m+1$ then $y_{1}, \ldots, y_{m}$ are the outputs of the pixel in the good state while $y_{m+l}, \ldots, y_{n}$ are the outputs of the pixel in the defective state. Assuming a constant failure rate, then

$$
\operatorname{Prob}\left(y_{k+1}=h o t \mid y_{k}=\operatorname{good}\right)=q \text {. }
$$

For each pixel, given the output reading from each image, we can evaluate the likelihood of the pixel output being good or defective based on the information from the neighboring pixels using Equations (3) and (4). By comparing pixel reading with a constant failure mode, the maximum likelihood function is represented by Equation (8), where both the failure rate $q$ and the first defective image $k=m+1$ are unknown.

$$
\begin{gathered}
L(m, q)=\prod_{k=1}^{m} G\left(y_{k}\right) \prod_{k=m+1}^{n} H\left(y_{k}\right) \times(1-q)^{m-1} q \\
q=\frac{1}{m}, m=1,2, \ldots, n-1
\end{gathered}
$$

The likelihood function will find both $m$ and $q$ for which the function $L(m, q)$ maximized. The corresponding $m$ for the maximized $L$ will be the last image for which the pixel is good; thus $m+l$ will be the index of the first defective image.

The main challenge when implementing the maximum likelihood technique is the amount of storage required. For a camera with $d$ defects, and a collection of $n$ images, we need to update the computation of the function $L$ for each defect. Since we are maximizing $L$ for $m=1$ to $n-1$, this will require an array of size $d \times(n-1)$. In addition, each pixel is composed of three colors R, G, B, thus we need to compute each color channel separately; thus the total required storage is $d \times(n-1) \times 3$ as shown in Figure 9. With a large collection of images (i.e., $n>100$ ), the storage space can easily exceed our system limit. Also, with new images added to the dataset, we must repeat the analysis from the first image. Our ultimate goal is to be able to continuously add new images and update the defect growth data with new defects without re-analyzing all images from the beginning. To perform a progressive analysis without additional storage, we

\begin{tabular}{|c|c|c|c|c|c|c|c|}
\hline$k^{\text {th }}$ image & 1 & 2 & 3 & 4 & 5 & $\ldots$ & $\mathbf{n}$ \\
\hline $\boldsymbol{m}=1: \quad L(1, q)$ & $\mathrm{G}\left(y_{l}\right)$ & $\mathrm{H}\left(y_{2}\right)$ & $\mathrm{H}\left(y_{3}\right)$ & $\mathrm{H}\left(y_{4}\right)$ & $\mathrm{H}\left(y_{5}\right)$ & $\ldots$ & $\mathrm{H}\left(y_{n}\right)$ \\
\hline $\boldsymbol{m}=2: \quad L(2, q)$ & $\mathrm{G}\left(y_{l}\right)$ & $\mathrm{G}\left(y_{2}\right)$ & $\mathrm{H}\left(y_{3}\right)$ & $\mathrm{H}\left(y_{4}\right)$ & $\mathrm{H}\left(y_{5}\right)$ & $\ldots$ & $\mathrm{H}\left(y_{n}\right)$ \\
\hline $\boldsymbol{m}=3: \quad L(3, q)$ & $\mathrm{G}\left(y_{1}\right)$ & $\mathrm{G}\left(y_{2}\right)$ & $\mathrm{G}\left(y_{3}\right)$ & $\mathrm{H}\left(y_{4}\right)$ & $\mathrm{H}\left(y_{5}\right)$ & $\ldots$ & $\mathrm{H}\left(y_{n}\right)$ \\
\hline$\vdots$ & & & & & & & $\vdots$ \\
\hline $\boldsymbol{m}=\boldsymbol{n}-1: \quad L(n-1, q)$ & $\mathrm{G}\left(y_{l}\right)$ & $\mathrm{G}\left(y_{2}\right)$ & $\mathrm{G}\left(y_{3}\right)$ & $\mathrm{G}\left(y_{4}\right)$ & $\mathrm{G}\left(y_{5}\right)$ & $\ldots$ & $\mathrm{H}\left(y_{n}\right)$ \\
\hline
\end{tabular}
first expand $G\left(y_{k}\right)$ and $H\left(y_{k}\right)$ terms used in Equation (8) as shown in Table 2.

Table 2. Maximum Likelihood function expansion 
Observing the above table, we know that for the $2^{\text {nd }}$ image, the computation of $L(1, q)$ is different by one term (i.e., $\left.H\left(y_{2}\right)\right)$. To reduce the amount of storage, we can compare the values of $L(1, q)$ and $L(2, q)$, and denote the larger one by $M A X$. Then, for the $3^{\text {rd }}$ image, we can compare $M A X \times H\left(y_{3}\right)$ with $L(3, q)$, and we update $M A X$ with the larger value among the two. In general, to perform a progressive computation without large memory storage we introduce two variables $M A X$ and $P R O G$, and for each new image, these two variables are updated according to Equations (9) and (10). The progressive search is summarized in Figure 10.

$$
\begin{gathered}
\left.M A X=\max \left(M A X \times H\left(y_{m}\right) \times(1-q)^{m-1} \times q, P R O G \times G\left(y_{m}\right) \times(1-q)^{m-1} \times q\right)\right) \\
P R O G=P R O G \times G\left(y_{m}\right)
\end{gathered}
$$

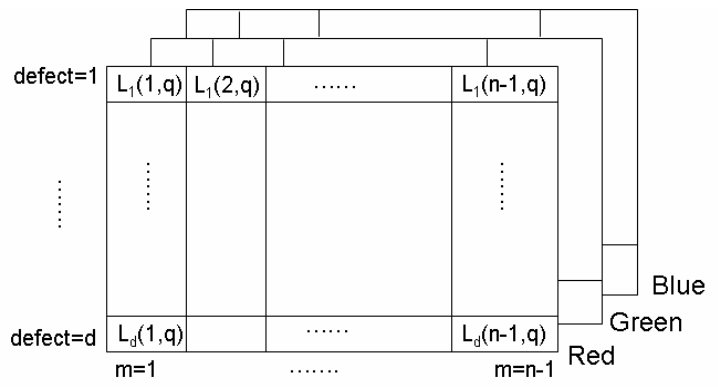

Figure 9. Maximum likelihood computation space.

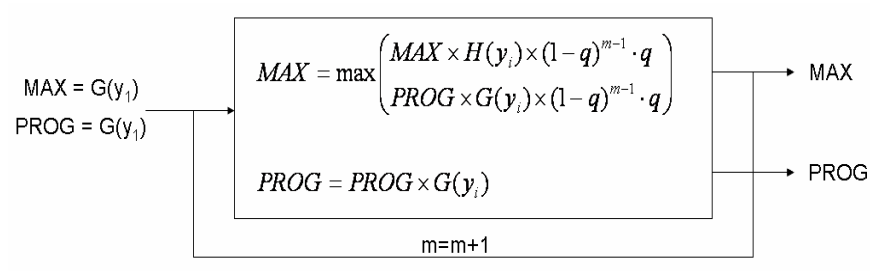

Figure 10. Maximum likelihood progressive computation.

\section{SIMULATION RESULTS}

In our previous study ${ }^{1}$ we have simulated the Bayes detection algorithm. To compare the accuracy of the detection of the Bayes and Maximum detection algorithms, a similar simulation test has been performed using the Maximum likelihood technique. To this end, we first need to model a sensor with $W \times H$ pixels and $d$ simulated hot pixels over the sensor area. Each simulated hot pixel is characterized by Equation (2) with a unique dark current value. At the beginning of the simulation, we start with a set of defect-free raw images and after $m$ images one defect is injected, where $m$ is different for each defect. Each raw image is converted to full color image using bilinear interpolation; thus we will observe defect cluster similar to Figure 5 in color images. By applying the Maximum likelihood function to each defect, we solve for $m$ and compare the calculated value with the known one.

The appearance of hot pixels is affected both by the dark parameter and exposure setting. To test the accuracy and limitation of both the Bayes and Maximum likelihood detection schemes, we simulate various dark current levels, exposure durations and interpolation schemes. The dark offset is a function of the exposure setting; thus, for images taken at short exposure, hot pixels are undetectable. Because most images are captured at below $0.8 \mathrm{~s}$ exposure, the detection algorithm must be able to detect defects in such short exposure images.

In the simulation, we use picture dataset from a 6MP sensor model and added 10 simulated hot pixels distributed randomly over the sensor area. By applying the Maximum likelihood function to each defect, the first defect date is defined by the $(m+1)$ image in the sequence. The detection error is the difference between the known $m$ and the value obtained by the detection algorithm. In the first set of simulations, we tested the detection accuracy for a set of images captured at the same exposure setting. The dark current magnitude varied between $(0.2-0.8 / \mathrm{s})$, and has been repeated for $3 \times 3,5 \times 5$ and $7 \times 7$ averaging for pixel estimation. The results of our simulations are summarized in Tables 3 and 4 . 
Table 3: Maximum likelihood detection performance at fixed exposure setting.

\begin{tabular}{|c|c|c|c|c|c|c|c|c|c|}
\hline \multirow{3}{*}{ Exposure(s) } & \multicolumn{3}{|c|}{$3 \times 3$ averaging } & \multicolumn{3}{|c|}{$5 \times 5$ ring averaging } & \multicolumn{3}{|c|}{$7 \times 7$ ring averaging } \\
\hline & \multirow{2}{*}{ \% Hit } & \multicolumn{2}{|c|}{ Error } & \multirow{2}{*}{$\%$ Hit } & \multicolumn{2}{|c|}{ Error } & \multirow{2}{*}{$\%$ Hit } & \multicolumn{2}{|c|}{ Error } \\
\hline & & mean & $\max$ & & mean & $\max$ & & mean & $\max$ \\
\hline 0.06 & 3.33 & 20 & -- & 10.00 & 6 & -- & 6.67 & 15 & -- \\
\hline 0.125 & 46.67 & 3 & -- & 43.33 & 3 & 1 & 43.33 & 5 & 1 \\
\hline 0.25 & 76.67 & 1 & 9 & 73.33 & 2 & 0 & 70.00 & 2 & 0 \\
\hline 0.5 & 90.00 & 0 & 1 & 93.00 & 0 & 1 & 90.33 & 0 & 1 \\
\hline
\end{tabular}

Table 4: Bayes detection performance at fixed exposure setting.

\begin{tabular}{|c|c|c|c|c|c|c|c|c|c|}
\hline \multirow{3}{*}{ Exposure(s) } & \multicolumn{3}{|c|}{$3 \times 3$ averaging } & \multicolumn{3}{|c|}{$5 \times 5$ ring averaging } & \multicolumn{3}{|c|}{$7 \times 7$ ring averaging } \\
\hline & \multirow{2}{*}{$\%$ Hit } & \multicolumn{2}{|c|}{ Error } & \multirow{2}{*}{ \% Hit } & \multicolumn{2}{|c|}{ Error } & \multirow{2}{*}{$\%$ Hit } & \multicolumn{2}{|c|}{ Error } \\
\hline & & mean & $\max$ & & mean & $\max$ & & mean & $\max$ \\
\hline 0.06 & 6.67 & 6 & -- & 33.33 & 5 & -- & 13.33 & 13 & -- \\
\hline 0.125 & 33.33 & 1 & -- & 43.33 & 2 & 10 & 53.33 & 1 & 36 \\
\hline 0.25 & 63.33 & 0 & 9 & 70.00 & 0 & 4 & 60.00 & 0 & 3 \\
\hline 0.5 & 70.00 & 0 & 1 & 83.33 & 0 & 3 & 73.33 & 0 & 3 \\
\hline
\end{tabular}

As we see in the simulation results, both the Bayes and the Maximum Likelihood detection schemes are able to identify all hot pixels in images taken at long exposures. Both detection algorithms show that the $5 \times 5$ interpolation achieves the best accuracy, with Bayes achieving a 83.3\% perfect defection and Maximum Likelihood achieving 93\%. The output level of the hot pixels is directly related to the exposure setting; at short exposures, the dark offset is also reduced. Due to spreading of defects by the demosaicing which affects the $3 \times 3$ averaging and the large interpolation error in the $7 \times 7$ ring averaging, it is not surprising to see the large averaging detection errors of these interpolation schemes at short exposures. Although neither detection scheme achieves high detection accuracy in short exposure images, Bayes detection displays significantly higher accuracy with 33\% perfect detection compared to the Maximum Likelihood detection of which only $10 \%$ of defect dates were correctly identified. In fact, over $50 \%$ of the defects in short exposure images were not detected by the Maximum Likelihood technique suggesting that this detection scheme can only operate on images captured at longer than $0.06 \mathrm{~s}$ exposure setting.

In the second set of simulations, we injected a set of defects with the same dark current magnitude for images captured with exposure range from $0.06 \mathrm{~s}$ to $0.5 \mathrm{~s}$. Here too we applied the Bayes and Maximum Likelihood detection algorithms. The results of the simulation are summarized in Tables 5 and 6 .

Table 5: Maximum likelihood detection performance at fixed dark current magnitude.

\begin{tabular}{|c|c|c|c|c|c|c|c|c|c|}
\hline \multirow{3}{*}{$\begin{array}{c}\text { Dark current } \\
(1 / \mathrm{s})\end{array}$} & \multicolumn{3}{|c|}{$3 \times 3$ averaging } & \multicolumn{3}{|c|}{$5 \times 5$ ring averaging } & \multicolumn{3}{|c|}{$7 \times 7$ ring averaging } \\
\hline & \multirow{2}{*}{$\%$ Hit } & \multicolumn{2}{|c|}{ Error } & \multirow{2}{*}{ \% Hit } & \multicolumn{2}{|c|}{ Error } & \multirow{2}{*}{$\% \mathrm{Hit}$} & \multicolumn{2}{|c|}{ Error } \\
\hline & & mean & $\max$ & & mean & $\max$ & & mean & $\max$ \\
\hline 0.2 & 60.00 & 1 & 7 & 56.67 & 2 & 11 & 36.67 & 4 & 18 \\
\hline 0.4 & 73.33 & 0 & 2 & 70.00 & 0 & 3 & 70.00 & 0 & 2 \\
\hline 0.6 & 80.00 & 0 & 4 & 86.67 & 0 & 2 & 83.33 & 0 & 3 \\
\hline 0.8 & 90.00 & 0 & 1 & 90.67 & 0 & 1 & 93.33 & 0 & 1 \\
\hline
\end{tabular}


Table 6: Bayes detection performance at fixed dark current magnitude.

\begin{tabular}{|c|c|c|c|c|c|c|c|c|c|}
\hline \multirow{3}{*}{$\begin{array}{c}\text { Dark current } \\
(1 / \mathbf{s})\end{array}$} & \multicolumn{3}{|c|}{$3 \times 3$ averaging } & \multicolumn{3}{|c|}{$5 \times 5$ ring averaging } & \multicolumn{3}{|c|}{$7 \times 7$ ring averaging } \\
\hline & \multirow{2}{*}{$\%$ Hit } & \multicolumn{2}{|c|}{ Error } & \multirow{2}{*}{$\%$ Hit } & \multicolumn{2}{|c|}{ Error } & \multirow{2}{*}{$\%$ Hit } & \multicolumn{2}{|c|}{ Error } \\
\hline & & mean & $\max$ & & mean & $\max$ & & mean & $\max$ \\
\hline 0.2 & 46.67 & 2 & 29 & 50.00 & 2 & 30 & 23.33 & 7 & 50 \\
\hline 0.4 & 65.00 & 1 & 10 & 70.00 & 3 & 5 & 73.33 & 0 & 1 \\
\hline 0.6 & 67.50 & 0 & 2 & 70.00 & 0 & 1 & 76.67 & 0 & 1 \\
\hline 0.8 & 80.00 & 0 & 1 & 82.50 & 0 & 1 & 73.33 & 1 & 33 \\
\hline
\end{tabular}

Our preliminary results suggest that both the Bayes and Maximum Likelihood detection schemes are able to detect all hot pixels with magnitude $>0.2 / \mathrm{s}$. Again, $5 \times 5$ ring averaging achieved the best detection accuracy in both detection schemes with $50-90 \%$ of defects dates identified accurately. Although the $7 \times 7$ ring averaging also achieved good detection accuracy, for missed detections it had one of the largest errors. Since $3 \times 3$ averaging uses the closest neighboring pixels estimation, demosaicing is most likely to distort these pixel locations in the present of a defect. In our detection, simple bilinear interpolation is used, thus the spreading of defects is not as significant. However, in reality, with a more complex demosaicing scheme, defect spreading can be more severe. For these simulations we only considered hot pixels with zero offset. In reality, $50 \%$ of the hot pixels we have identified have an offset, and because these offsets enhance the visibility of hot pixels, this will clearly enhance the detection accuracy.

Based on the simulation results of the two detection schemes, we have plotted the hit rate in Figure 10, for each experiment using a $5 \times 5$ ring averaging pixel estimation.

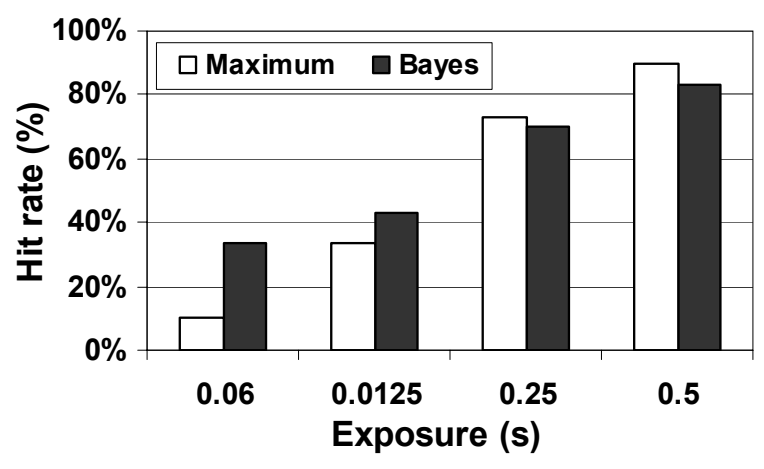

(a) Fixed exposure setting

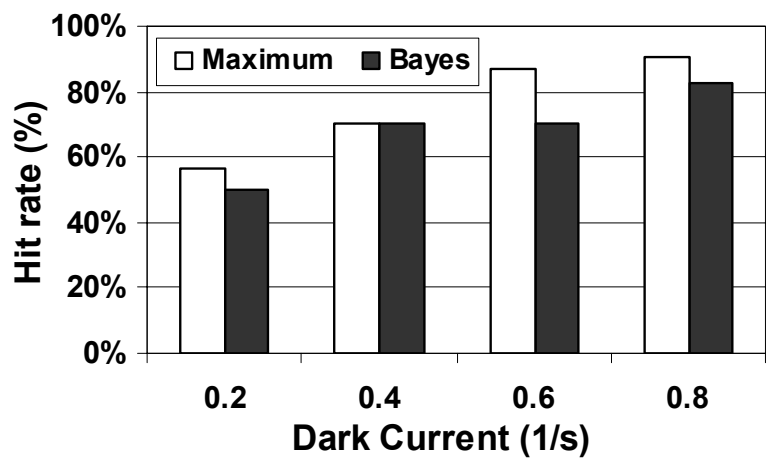

(b) Fixed dark current magnitude

Figure 10. Detection performance comparison at (a) fixed exposure setting, (b) fixed dark current magnitude.

Although the Maximum Likelihood detection algorithm achieves better detection accuracy, it is not suitable for a real image dataset because it suffers from low hit rates in short exposure images. Long exposure images are not common due to the difficulty in stabilizing the camera when the shutter opens for a long period of time. In fact, in our image data collection, less than $30 \%$ of the images were captured with exposures over $0.06 \mathrm{~s}$. Furthermore often several cameras have options that automatically do a background subtraction to remove some hot pixel effects for exposures $>\sim 0.8 \mathrm{sec}$, reducing this data set even further. The accumulation nature of the Bayes detection algorithm allows small changes in $G\left(y_{i}\right)$ and $H\left(y_{i}\right)$ to be reflected in the accumulated statistics, thus defects are identified even in short exposure images. At the same time, the Bayesian detection can suffer from false detections caused by small marginal interpolation errors. On the other hand, Maximum Likelihood detection depends on the discrepancy between $G\left(y_{i}\right)$ and $H\left(y_{i}\right)$ which is not significant enough in short exposure images where the dark offset is minimal. Although Bayes detection suffers from false defection due to inaccurate pixel estimation, previous proposed local statistics correction ${ }^{2}$ had proven to eliminate these detection errors. A comparison between enhanced Bayes detection will be presented in future work and modification of the Maximum Likelihood detection technique will be explored to expand the exposure range for this algorithm. 


\section{EXPERIMENTAL RESULTS ON ACTUAL CAMERA DATA}

In a recent $s_{\text {sudy }}{ }^{2}$, we tested the Bayes detection on a real image data set and found good ability to find the occurrence date of actual hot pixels defects. With our expanding data collection, we applied the Bayes detection algorithm to find the defect growth characteristics of four cameras with a cumulative total of 35 hot pixels. From our previous test results on three cameras, we know that the $5 \times 5$ interpolation achieves the closest detection to our visual inspected detection; therefore, we applied the Bayes detection using the $5 \times 5$ ring averaging. For each camera we first identify defect dates for individual fault using both visual inspection and Bayes detection. To reduce large error in Bayes detection due to inaccurate pixel estimation we corrected any false detection using the local-statistics procedure discussed in Section 3.2. The identified defect date for each camera is plotted against the camera age as shown in Figure 11, and a linear fit is used to estimate the defect growth rate. The resulting defect growth rates for each camera from visual inspection and Bayes detection are summarized in Table 7. From this experiment, we can conclude that the Bayes detection technique is able to closely approximate the visually inspected defect growth rate. In fact, the worst approximation has less than $3 \%$ error compared to the visual inspection rate.

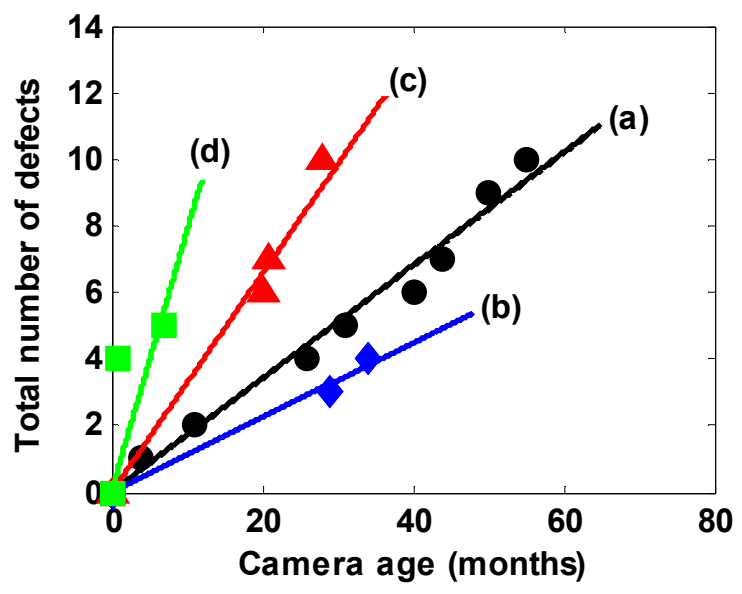

Figure 11. Defect Growth results from 4 cameras.

Table 7. Visual Inspection and Bayes detection growth rate comparison.

\begin{tabular}{|l|c|c|c|c|}
\hline & $\begin{array}{c}\text { Sensor } \\
\text { Type }\end{array}$ & $\begin{array}{c}\text { Visual Inspected defect growth rate } \\
\text { (defects / year) }\end{array}$ & $\begin{array}{c}\text { Bayes detected defect growth rate } \\
\text { (defects / year) }\end{array}$ & error \\
\hline Camera A & APS & 2.04 & 2.03 & $0.5 \%$ \\
Camera B & APS & 1.34 & 1.34 & $0.0 \%$ \\
Camera C & CCD & 3.94 & 4.04 & $3.0 \%$ \\
Camera D & CCD & 9.36 & 9.36 & $0.0 \%$ \\
\hline
\end{tabular}

The results from all four cameras displayed a common characteristic where all imagers exhibited a linear gradual growth of defects, indicating that defects are not caused by sudden shock of the camera or fabrication failure. In fact, the similar behavior of the two sensor technologies indicates that defects are caused by a similar external source. Knowing the sensor type for each tested camera, our results suggest that CCD sensors develop defects at a much higher rate then APS cameras, which is similar to our observation in Section 2. The highest defect rate among these four imagers is camera D where we estimate a growth rate of 9.36 defects/year. In fact, multiple faults were found at the early age of this camera, where we observed a few defects that have developed in a short time period. It was clear that these defects developed in the field and are not manufacture time defects. However, these defects possibly occurred during the shipment flight from the factory, where the camera has been exposed to a high radiation environment. 


\section{CONCLUSION}

All digital imagers develop defects in the field and inability to recognize the increasing presence of in-field defects can cause the image quality to degrade over time. By analyzing the characteristics of in-field defects, we can confirm that these defects are not related to material degradation or a single event. From laboratory calibration, we can identify both defect location and behavioral parameters. However, to trace the development of defects we must analyze the full historical image data set to determine the first appearance of each defect. Although we can visually inspect for defects in each image, this process is slow and allows limited access to large data sets o cameras. Therefore, we have developed two detection algorithms: a Bayesian accumulation scheme and a Maximum likelihood scheme. Monte Carlo simulation on both algorithms showed that both were able to identify $80 \%$ of the defect dates accurately. In fact, the Maximum likelihood detection was able to achieve a $90 \%$ hit rate for detection in long exposure images. The main drawback of the Maximum likelihood detection is that only $10 \%$ of defects were detectable in short exposure images $(<0.06 \mathrm{~s})$, as compared to a $40 \%$ hit rate with Bayesian detection. As majority of images are captured at short exposure setting, additional refinement is needed to increase the detection accuracy of the Maximum likelihood detection in the short exposure range.

Refinement with local statistics on the Bayesian detection allows this detection scheme to achieve sufficient accuracy when analyzing real image datasets. Application of the Bayes detection on image datasets from four cameras (2 APS, 2 $\mathrm{CCD}$ ) indicated that this detection scheme was able to identify the defect growth rate with less than $3 \%$ difference from the visual inspection. Preliminary detection results suggest that APS imagers develop 1-2 defects/year whereas CCD imagers develop 4-9 defects/years. The large discrepancy in defect growth rate between the two sensors suggests that CCD sensors might be more sensitive to cosmic ray radiation.

\section{REFERENCES}

[1] J. Leung, J. Dudas, G.H. Chapman, Z. Koren, and I. Koren, "Characterization of defect development During Digital Imager Lifetime,” Proc. Electronic Imaging, Sensors, Cameras, and Systems for Industrial/Scientific Application IX, Vol. 6826, San Jose, Jan. 2008.

[2] J. Leung, G. Chapman, I. Koren, and Z. Koren, "Automatic Detection of In-field Defect Growth in Image Sensors," Proc. of the 2008 IEEE Intern. Sympos. on Defect and Fault Tolerance in VLSI Systems, pg. 220-228, Oct. 2008.

[3] J. Leung, J. Dudas, G. H. Chapman, I. Koren, Z. Koren, "Quantitative Analysis of In-Field Defects in Image Sensor Arrays," Proc. of the 2007 Intern. Symposium on Defect and Fault Tolerance in VLSI, pg. 526-534, Rome, Italy, Sept 2007.

[4] A. J.P. Theuwissen, "Influence of Terrestrial Cosmic Rays on the Reliability of CCD Image Sensors," IEDM 2005, San Francisco, CA, 2005. 